\title{
Gemini/IFU Observations of Galactic Outflows in Starburst Galaxies
}

\author{
Linda J. Smith ${ }^{1,2}$ and Mark S. Westmoquette ${ }^{2}$ \\ ${ }^{1}$ Space Telescope Science Institute and European Space Agency, 3700 San Martin Drive, \\ Baltimore, MD 21218, USA \\ ${ }^{2}$ Department of Physics and Astronomy, University College London, Gower St., London \\ WC1E 6BT, UK
}

\begin{abstract}
We present Gemini/IFU observations that sample the roots of the galactic wind outflows in the starburst galaxies NGC 1569 and M82. The good spatial and spectral resolutions of these observations allow us to probe the interactions of cluster winds with their environments on small scales. For both galaxies, we find a ubiquitous broad (200-300 $\left.\mathrm{km} \mathrm{s}^{-1}\right) \mathrm{H} \alpha$ component underlying a brighter narrower component. By mapping the properties of the individual line components, we find correlations that suggest that the broad component results from powerful cluster wind-gas clump interactions. For NGC 1569, there is little evidence for organised gas flows within the central zone and we suggest that the flow-dominated wind must form well beyond the region containing the massive star clusters. For M82, we find that the kinematics of the wind base are very complex; the width of the broad component reaches values of $>350 \mathrm{~km} \mathrm{~s}^{-1}$ at the base of the wind, and the outflow itself rapidly becomes chaotic in the inner wind region.
\end{abstract}

Keywords. ISM: jets and outflows - ISM: kinematics and dynamics - galaxies: ISM - galaxies: starburst

\section{Introduction}

Massive stars feed back energy into the interstellar medium (ISM) via stellar winds and supernova explosions. This feedback mechanism is thought to play a fundamental role in the formation and evolution of galaxies. In starburst galaxies, where star formation is intense and young massive star clusters are formed, galaxy-wide superwinds are seen. The onset and development of these outflows can be studied by observing local starburst galaxies. In particular, we wish to understand how and where the individual winds from star clusters interact with each other, and combine to produce large-scale outflows. Past studies of galactic outflows have tended to concentrate on their large-scale aspects; here we present a summary of our recent work aimed at understanding the small-scale characteristics of the cluster wind flows in the two nearby starburst galaxies NGC 1569 and M82.

\section{NGC 1569}

NGC 1569 is a nearby $(2.2 \pm 0.6 \mathrm{Mpc})$ dwarf irregular galaxy that has recently undergone a galaxy-wide burst of star formation that peaked between $100 \mathrm{Myr}$ and 5-10 Myr ago (Greggio et al. 1998). The two well-known super star clusters (SSCs) A and B are prominent products of the starburst episode, and probably dominate the energetics of the central region. An extended system of $\mathrm{H} \alpha$ filaments, indicative of a bipolar outflow is seen (Heckman et al. 1995; Martin 1998), and X-ray observations show that the wind emanates from the full extent of the $\mathrm{H} \alpha$ disk (Martin, Kobulnicky \& Heckman 2002). 


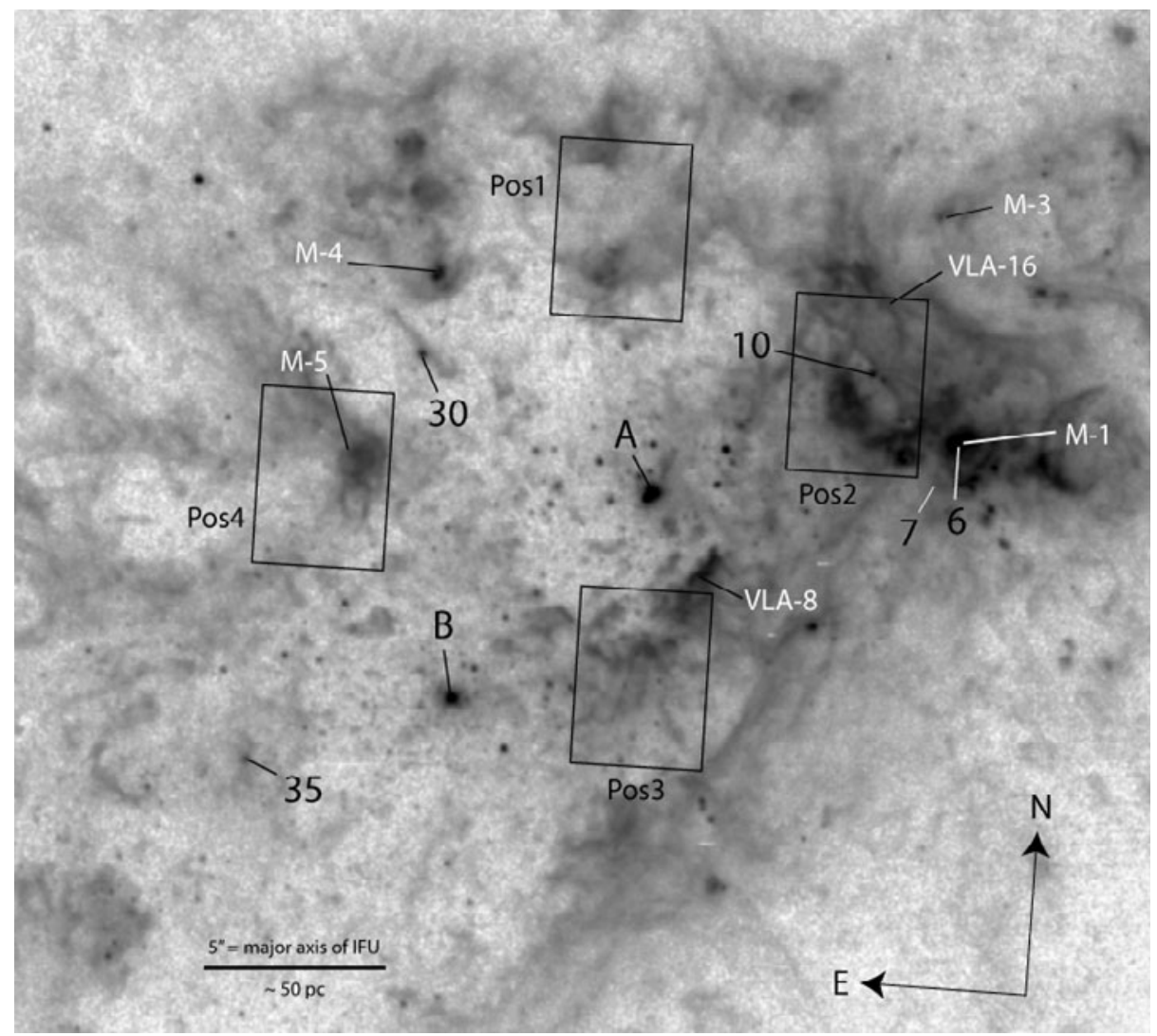

Figure 1. HST/WFPC2 F656N image of the central region of NGC 1569 showing the positions of the four IFU fields. A number of the most prominent star clusters (Hunter et al. 2000) and radio continuum sources (Greve et al. 2002) are labelled in black and white respectively.

\subsection{Observations and Analysis}

To probe the roots of the wind outflow from NGC 1569, we obtained observations of four regions near the centre of the galaxy with the Gillett Gemini North 8.1-metre telescope and the Gemini Multi-Object Spectrograph (GMOS) Integral Field Unit (IFU; AllingtonSmith et al. 2002). In Fig. 1, we show the positions of the four IFU fields on an archive Hubble Space Telescope (HST) F656N image obtained with the Wide Field Planetary Camera 2 (WFPC2). The GMOS/IFU consists of 500 object fibres (each of diameter 0.2 arcsec) arranged in a rectangular array of size $5 \times 3.5 \operatorname{arcsec}^{2}\left(\right.$ or $50 \times 35 \mathrm{pc}^{2}$ for NGC 1569). Our chosen spectral setup gave us a wavelength coverage of 4740-6860 $\AA$ with a dispersion of $0.34 \AA \mathrm{pix}^{-1}$. The data were reduced using the standard Gemini pipeline; procedures included flat-fielding, cosmic-ray cleaning, sky subtraction, throughput correction, flux calibration and differential atmospheric correction. Full details can be found in Westmoquette et al. $(2007 \mathrm{a}, \mathrm{b})$.

For each IFU position, we have 500 spectra covering the important nebular diagnostic emission lines of [O III], $\mathrm{H} \beta,[\mathrm{N}$ II], $\mathrm{H} \alpha$ and [S II] . The signal-to-noise and spectral resolution (60-75 $\left.\mathrm{km} \mathrm{s}^{-1}\right)$ of these data are sufficiently high to resolve multiple Gaussian components to each emission line. In order to quantify and compare the properties of the ionized gas, we have fitted multiple Gaussian components to all of the spectral line profiles. To do this, we used an interactive $\chi^{2}$ minimisation curve-fitting program written 

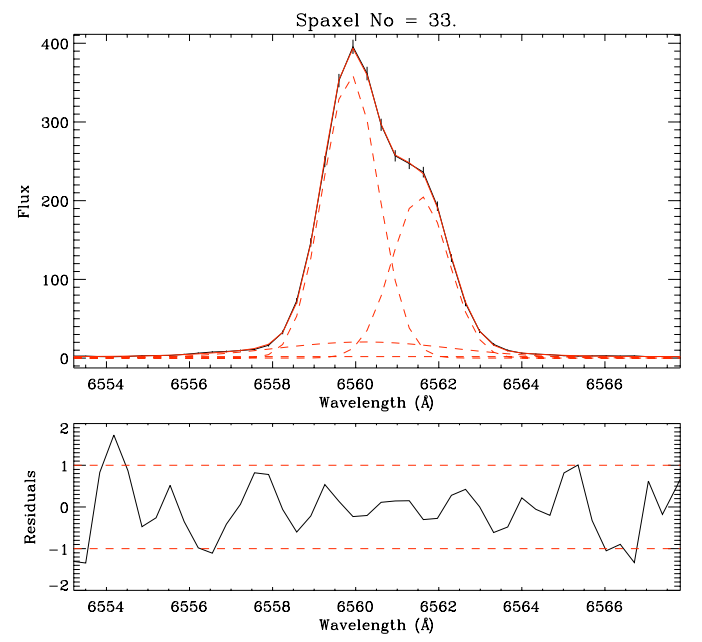

Figure 2. Example of a three component Gaussian fit to the $\mathrm{H} \alpha$ emission line profile for one spaxel in IFU position 1 . The solid line is the observed line profile and the dashed lines represent the individual Gaussian fits. The normalised residuals to the fit are shown below.

in IDL (PAN; Dimeo 2005) to automate the line profile fitting. To determine how many Gaussian components best fit an observed profile, we applied the statistical F-test to the $\chi^{2}$ ratios, together with a number of additional physical tests and filters (see Westmoquette et al. (2007a) for a detailed description of our fitting procedure and its associated uncertainties). Decomposing each compound line profile meant that we could analyse the properties of each component separately, and this allowed us to disentangle flux, velocity and line width information.

In general, we find the line profiles to be composed of a bright, narrow component (hereafter C1) overlaid on fainter, broad emission (hereafter C2). We find bright C1 emission across all four regions, well characterised by a Gaussian function with a distinct lower limit to its FWHM of $\sim 35 \mathrm{~km} \mathrm{~s}^{-1}$. We also measure a fainter line component (C2) over all four regions with line widths between FWHM $\sim 100-400 \mathrm{~km} \mathrm{~s}^{-1}$. The total radial velocity spread between these components is only $\sim 70 \mathrm{~km} \mathrm{~s}^{-1}$. We also identify a third emission line component in some regions which is of higher velocity and can be associated with expanding shells of gas. An example of a line profile showing all three components is shown in Fig. 2.

By plotting maps of the fit parameters as a function of spatial position, we find a number of correlations. In particular, we find that the width of the broad underlying emission (C2) is both highly correlated with the flux of the narrow component (C1) and anti-correlated with its own flux. The former of these two correlations is shown in Fig. 3, where we plot the $\mathrm{C} 1$ line flux in contours over the $\mathrm{C} 2$ line width for IFU position 2.

\subsection{Interpretation}

We first discuss the origin of the bright "narrow" $\mathrm{C} 1$ component. The integrated H I velocity dispersion for the disk of NGC 1569 is $\sigma \approx 15 \mathrm{~km} \mathrm{~s}^{-1}$ indicating that the neutral ISM is very disturbed (Mühle et al. 2005), presumably due to the combined effects of stellar winds and supernovae. We would thus expect the HiI gas in NGC 1569 to have similar or higher velocity dispersions to the H I. Indeed, we find that there is a distinct $\sim 35 \mathrm{~km} \mathrm{~s}^{-1}$ lower limit to the C1 FWHM. After correction for thermal broadening, this becomes $\sigma \approx 12 \mathrm{~km} \mathrm{~s}^{-1}$. Line widths of this order for the main ionized gas component have been observed in many young star-forming regions e.g. 30 Dor or 


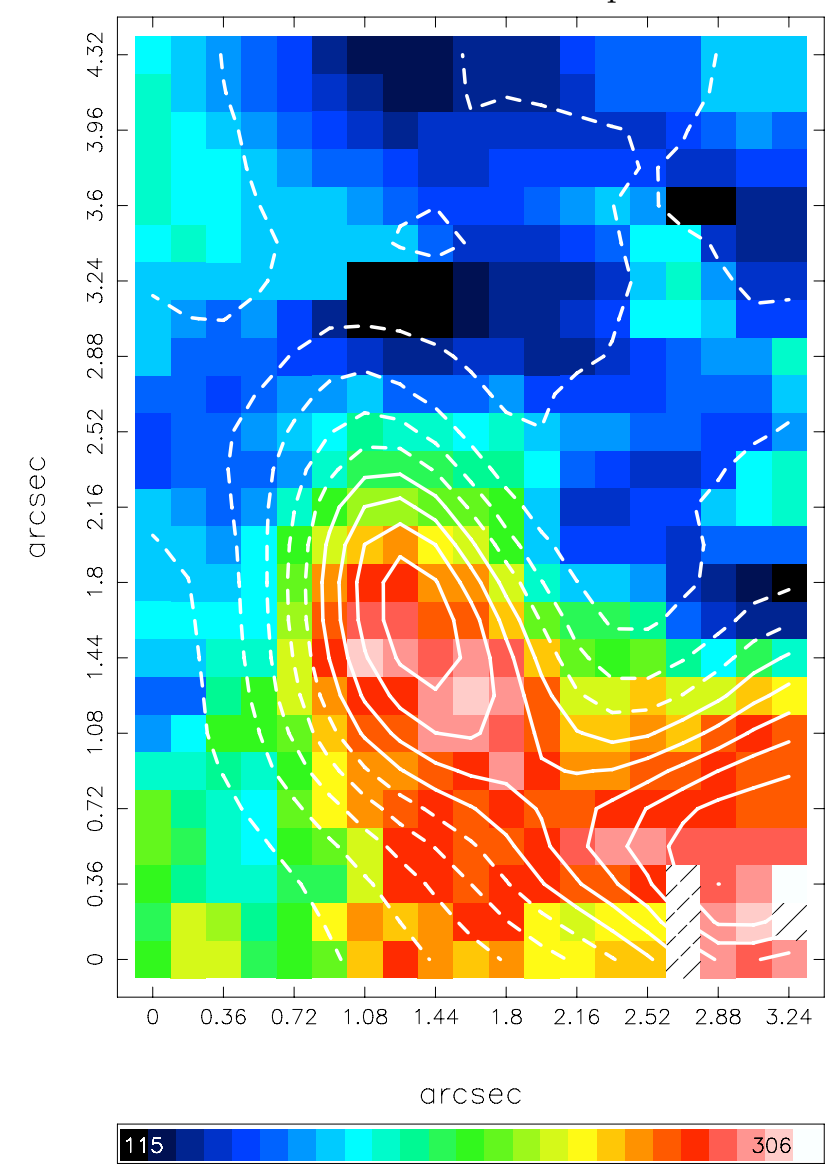

Figure 3. Contours of $\mathrm{C} 1$ flux overlaid on the C2 FWHM map for IFU position 2. Here the correlation between the bright $\mathrm{H} \alpha$ knots seen in $\mathrm{C} 1$ and the broadest $\mathrm{C} 2$ lines can be seen clearly.

NGC 604 (Chu \& Kennicutt 1994; Yang et al. 1996). Various explanations have been put forward to explain its width, including multiple unresolved expanding shells integrated along the line-of-sight, and gravitational broadening through virial motions. We find that unresolved expanding shells cannot contribute significantly to the observed widths of $\mathrm{C} 1$, since the point at which coherent shells break up is when their expansion velocity equals the ambient ISM turbulent velocity (i.e. the $\sim 35 \mathrm{~km} \mathrm{~s}^{-1}$ lower limit). Simple calculations for the expected level of gravitational broadening show that it may contribute a significant fraction of the observed width. Thus, we interpret $\mathrm{C} 1$ as representing the turbulent ISM of NGC 1569, where its motions (width) result from a convolution of the general stirring effects of the starburst and gravitational virial motions (thus giving rise to the clear FWHM lower limit). Additional minor contributions may result from unresolved expanding shell components along the line-of-sight.

C2 cannot result from any of the effects described above since none are able to produce such broad line widths, or such strong correlations between the emission line maps as shown for example in Fig. 3. However, we expect that C2 must somehow be associated with the starburst environment i.e. the interactions of high-energy photons and hot, fast-flowing winds from the nearby clusters with the cold, ambient ISM. An exchange of energy and mass between the hot and cold phases of the ISM can occur through: photoevaporation; conductively-driven thermal evaporation; hydrodynamic ablation; and 
turbulent mixing (for a review see Pittard (2007)). When the hot, tenuous medium is fast-flowing, as in the case of stellar or cluster winds, shearing between the high velocity flow and the cloud surface can set up a turbulent mixing layer that can drive further evaporation. The impacting wind can also physically strip or ablate material off the clump surface, and entrain it into the flow (Scalo 1987).

Begelman \& Fabian (1990) described a model for a turbulent mixing layer that forms between the hot, inter-cloud gas and the surface layers of cool or warm clouds in nearpressure equilibrium. The UV radiation emitted from the mixing layer photoionizes the cold gas in the cloud surface giving rise to optical emission lines with characteristic velocities of a fraction of the hot phase sound speed. Slavin et al. (1993) expanded on this idea by modelling the cloud interface in detail and considering the hot gas flow. Their model predicts strong optical [O III] and $\mathrm{H} \alpha$ emission with non-Gaussian (broad-winged) line profiles caused by the high levels of turbulence, clearly in good agreement with what we observe.

We therefore conclude the most likely explanation of the origin of $\mathrm{C} 2$ is emission from a turbulent mixing layer formed at the surface of dense clouds resulting from the viscous coupling between the cool clump material and the hot, fast, winds from the surrounding clusters. Material is also removed from this layer through thermal evaporation and/or mechanical ablation, resulting in the mass-loading of the flow. Our observations suggest that this mass-loading occurs close to or within the star-forming disk. In addition, we find from the lack of significant emission line velocity variations, that there is little evidence for organised gas flows within the central zone of NGC 1569. The flow-dominated wind must therefore form well beyond the region containing the massive star clusters; the wind sonic point probably lies at a distance of $>100-200 \mathrm{pc}$ from the starburst.

\section{M 82}

Recently, we have obtained Gemini-North GMOS observations of the inner wind in the starburst galaxy M82 covering six IFU positions. The pointings were chosen to sample the roots of the wind close to the bright star-forming regions $\mathrm{A}$ and $\mathrm{C}$.

We find that the $\mathrm{H} \alpha$ emission line profiles are similar to those observed for NGC 1569 with a narrow bright component superimposed on a broad underlying component. While the broad component is present throughout the starburst clumps (with widths of $\sim 200 \mathrm{~km} \mathrm{~s}^{-1}$ ), it reaches maxima of $>350 \mathrm{~km} \mathrm{~s}^{-1}$ at the base of the wind outflow region, following the linear 'streamer' morphology seen in HST imaging. In many regions we also identify a second narrow component offset in velocity. The kinematics of the wind base are clearly very complex.

Furthermore, for the first time we are able to measure the gas density (via the [S II] ratio) in all three line components, and find that the distribution differs in each. The density in the main, bright, narrow component peaks to the north of both regions $\mathrm{A}$ and C (in agreement with Westmoquette et al. 2007c), however the densities found in the broad underlying component peak within the starburst clumps themselves.

By extracting position-velocity diagrams along the major axis and along parallel lines stepping out into the inner-wind, we are able to examine the gas kinematics in more detail. We find that the outflow rapidly becomes chaotic. Interestingly, the broad component follows a shallower rotation curve, meaning that the motion of this gas may be de-coupled from that of the gas emitting the bright, narrow component. Clear evidence for expanding structures become visible at distances $>3^{\prime \prime}$ from the major axis, with line-splitting of $>100 \mathrm{~km} \mathrm{~s}^{-1}$. 


\section{Acknowledgements}

We would like to thank and acknowledge our collaborators: Jay Gallagher, Katrina Exter (NGC 1569) and Jay Gallagher, Gelys Trancho, Nate Bastian and Iraklis Konstantopoulos (M82).

\section{References}

Allington-Smith, J., Murray, G., Content, R., et al. 2002, PASP, 114, 892

Begelman, M. C. \& Fabian, A. C. 1990, MNRAS, 244, 26P

Chu, Y.-H. \& Kennicutt, R. C. 1994, ApJ, 425, 720

Dimeo, R. 2005, PAN User Guide, ftp://ftp.ncnr.nist.gov/pub/staff/dimeo/ pandoc.pdf/

Greggio, L., Tosi, M., Clampin, M., et al. 1998, ApJ, 504, 725

Greve, A., Tarchi, A., Hüttemeister, S., et al. 2002, A\&SA, 381, 825

Heckman, T. M., Dahlem, M., Lehnert, M. D., et al. 1995, ApJ, 448, 98

Hunter, D. A., O'Connell, R. W., Gallagher, J. S., \& Smecker-Hane, T. A. 2000, AJ, 120, 2383

Martin, C. L. 1998, ApJ, 506, 222

Martin, C. L., Kobulnicky, H. A., \& Heckman, T. M., 2002, ApJ, 574, 663

Mühle, S., Klein, U., Wilcots, E. M., \& Hüttemeister, S., 2005, AJ, 130, 524

Pittard, J. M. 2007, in T. W. Hartquist, J. M. Pittard, S. A. E. G. Falle, (eds.), Diffuse Matter from Star Forming Regions to Active Galaxies. (Dordrecht: Springer), Astrophysics 8 Space Science 245

Scalo, J. M., 1987, in D. J. Hollenbach, H. A. Thronson, Jr, (eds.), Interstellar Processes, (Dordrecht: Reidel), Astrophys Space Sci Libray 134, 349

Slavin, J. D., Shull, J. M., \& Begelman, M. C. 1993, ApJ, 407, 83

Westmoquette, M. S., Exter, K. M., Smith, L. J., \& Gallagher, J. S., III, 2007a, MNRAS, 381, 894

Westmoquette, M. S., Smith, L. J., Gallagher, J. S., III, \& Exter, K. M. 2007b, MNRAS, 381, 913

Westmoquette, M. S., Smith, L. J., Gallagher, J. S., III, et al. 2007c, ApJ, 671, 358

Yang, H., Chu, Y.-H., Skillman, E. D., \& Terlevich, R. 1996, AJ, 112, 146

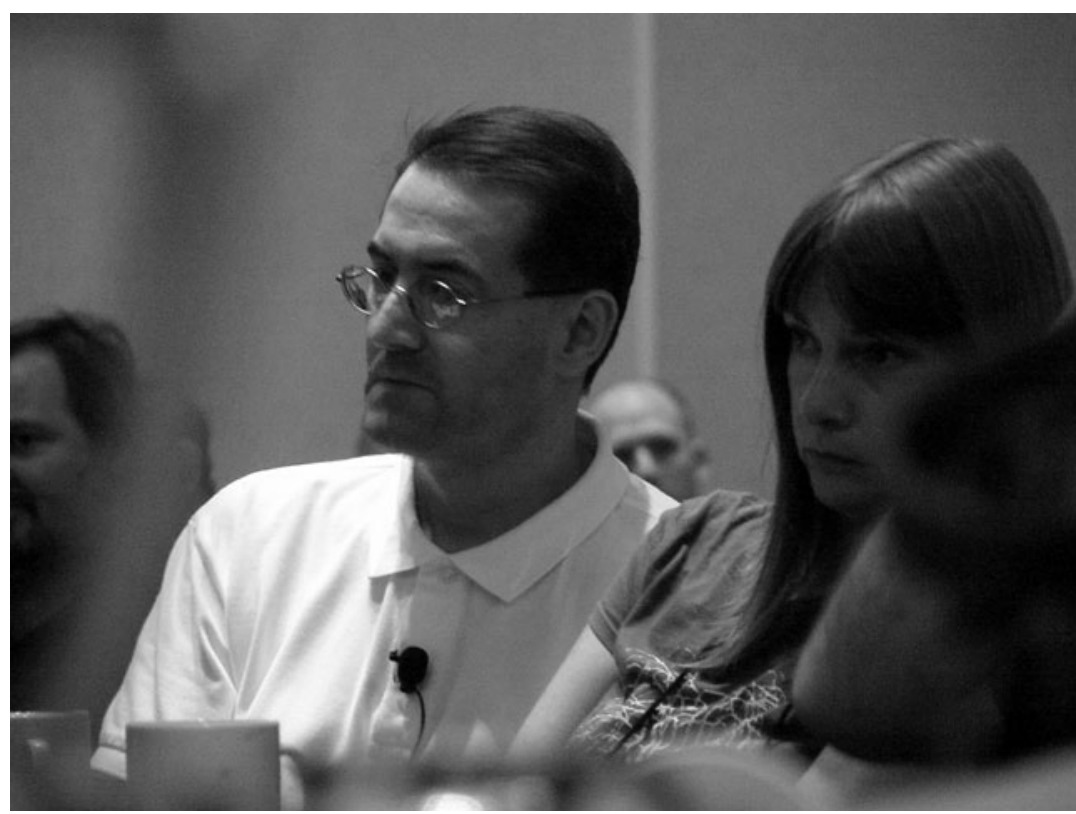

Claus Leitherer (left) and Linda Smith (right). 